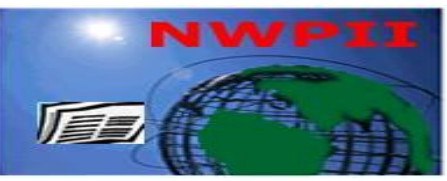

American Journal of Biomedical Sciences

ISSN: 1937-9080

nwpii.com/ajbms

\title{
Diethylentriaminepentaacetic Acid-deoxyglucoseamine (DTPA-DG): Novel Nanosized Anti-Wilson's Disease Cell Model
}

\author{
Mojgan Mashayekhi $^{1 \times}$, Massoud Amanlou ${ }^{1 \times}$, Kourosh Sadeghi ${ }^{2}$, Mona Mosayebniya ${ }^{1}$, \\ Mehdi Shafiee Ardestani ${ }^{1,3}$, Bita Mehravi ${ }^{4}$
}

${ }^{1}$ Department of Medicinal Chemistry, Faculty of Pharmacy, Tehran University of Medical Sciences, Tehran, Iran

${ }^{2}$ Department of Clinical Pharmacy (Pharmacotherapy), Faculty of Pharmacy, Tehran University of Medical Sciences, Tehran, Iran

${ }^{3}$ Department of Hepatitis and AIDS, Pasteur Institute of Iran, Tehran, Iran

${ }^{4}$ Medical Physics and Biomedical Engineering and Nanomedicine Department, Faculty of Medicine,

Shahid Beheshti Medical University, Tehran, Iran

${ }^{\times}$These authors are contributed equally to the experiments (Considered as 'First Author')

"Corresponding author:

Dr. Mehdi Shafiee Ardestani

Department of Hepatitis and AIDS

Pasteur Institute of Iran and Department of Medicinal Chemistry

Faculty of Pharmacy Tehran University of Medical Sciences

Tehran, Iran

Tel/Fax: +98-2166953311

Email: shafieeardestani@gmail.com

Received:17 May 2012;| Revised:18 November 2012; | Accepted: 5 December 2012

\begin{abstract}
Wilson disease (WD) is an autosomal recessive disorder well recognized by the copper progressive accumulation. Copper accumulation in various organs and tissues causes toxic effects in various tissues including liver, brain, kidneys and eyes. Chelation therapy is the mainstay of treatment for patients with Wilson's disease. Chelators are naturally occurring or chemically designed molecules that bind to specific toxins in the body and promote excretion of them. Diethylenetriamine penta-acetic acid (DTPA) is one of the well-known Chelators which is used in WD treatment but it's main defect is that it cannot enter into the intracellular space. When an amine such as D-glucosamine (DG) was conjugated to DTPA, the resulting compound obtains the ability to enter cells and may be used to treat the intracellular metal overload. Based on the hypothesis, A new compound consisting of D-glucose $(1.1 \mathrm{~nm})$ conjugated DTPA, was synthesized and evaluated on HepG2 WD cell model in vitro. Our results revealed that that the copper accumulation significantly $\mathrm{P}<0.05$ decreased in the HepG2 WD cells by DTPA-DG as compared with D-penicillamine (as gold standard treatment) compared with the D-penicillamine (gold standard). Interestingly, no toxicological findings were seen in HepG2 WD cell. Based on our findings, administration of DTPA-DG could be significantly decrease the copper accumulation in HepG2 WD cell model without any toxicity and seems to have a very good prognosis_for treatment of WD in the future.
\end{abstract}


Keywords: DTPA-DG, copper overload, Wilson's disease, chelator, cell uptake.

\section{Introduction}

Copper is one of the essential trace elements for many enzymatic systems in human body. While trace amounts of copper are needed to sustain life, excess copper is extremely toxic. [1]

Wilson disease (WD) is an autosomal recessive disorder of copper transport leading accumulation of copper in the liver and brain of affected individuals [1]. Copper accumulates in the cytosol of hepatocytes, leading to hepatic necrosis and release large amounts of copper into the bloodstream of large amounts of copper into the blood stream, causing damage to erythrocyte membrane and hemolytic anemia. Finally Copper accumulates in other vital organs such as the brain, kidneys, and cornea. Defective biliary excretion of copper may be the single most important cause of copper accumulation in Wilson disease [1].

Treatment methods for patients with Wilson's diseas can produce a negative overall copper balance. There are usually two phases of treatment for symptomatic WD patients: first, a chelating agent is administered to enhance systemic systemic depletion of metals by urinary excretion (i.e., D-penicillamine or TETA), Then either a chelating agent or zinc salts are administered to prevent accumulation or reaccumulation of copper to toxic levels [2].
The use of chelating agents to regulate metal ion/metalloid toxicity began within the early decade with researchers like Alfred the Great Werner, Paul Ehrlich, and Carl Voegtlin progressing to cut back toxicity of arsenic- and antimony-containing medicine that won't to treating parasitic infections like syph. Smallmolecule chelators were applied to alleviate the results of significant metal and nonmetallic overload. the employment of small-molecule chelators to alleviate accidental overexposure to metal ions began in 1941 with the (questionably appropriate) use of change state for lead intoxication [2].

Some of the chelating agents which have been successfully used to treat metal intoxication are shown in Fig. 1. British anti-Lewisite (BAL) was originally developed to treat arsenic toxicity, however its potential for treating hereditary disease was recognized by J.N. Cumings in 1948 . the necessity to search out Associate in Nursing oral different agent to BAL for treating Wilson's illness diode to the introduction of Dpenicillamine and trientine by Dr John Walshe in 1956 and 1969, severally. DMSA and DMPS ar less nephrotoxic and additional hydrophilic analogues of BAL, and ar accustomed treat mercury intoxication. EDTA and its congenator DTPA are often accustomed treat plumbism and ar effective in removing radionuclides [3].
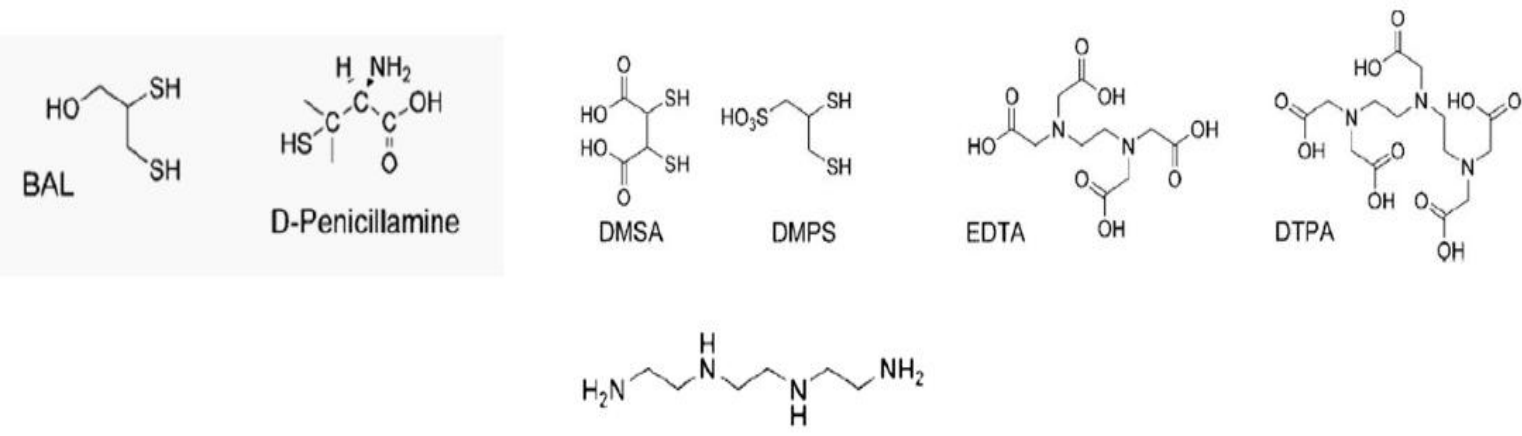

Trientine

Figure 1: Ligands developed from 1940-1970 to treat chronic and acute metan intoxication. 
DTPA is associate degree organic substance encompasses a diethylenetriamine backbone structure with 5 carboxymethyl teams. DTPA with its high affinity for metal cations like copper will kind reliable complexes. This chelator is poorly absorbed within the epithelial duct $(<5)$. Its volume of distribution is near living thing water volume, and this chelator is chop-chop excreted within the excretory product while not vital metabolism [4].

The major drawback with DTPA is it's curtail cellular uptake [5,6]. to enhance DTPA uptake by cells, varied structural derivatives are synthesized and tested toward elevating DTPAcopper advanced formation and cellular uptake [7]. several strategies are established for conjugation of DTPA to totally different chemical substances, however sophisticated synthesis method was a serious limiting issue. [7].

Glucosamine could be a scaffold for a glucosyl substance as a result of the methane series acts as a helpful target for additional functionalization. there's abundant proof within the literature counsel that $\mathrm{N}$-functionalized glucosamines show activity with aldohexose transporters and hexokinase enzymes [8-10].

When D-glucosamine (DG) is conjugating to DTPA, the resulting compound obtain the ability to the ability to enter cells, Accordingly the DTPA-DG may be used as a novel strategy for the diagnosis and/or WD treatment. An amino group at position 2 of the glucose moiety. DTPADG also has an amino group at this position in its glucose moiety [11-14].

In this paper, We describe a method for synthesis of DTPA-DG employing a high yield and few reaction steps- After that we designed some biological test on this compound chelating with intracellular copper accumulated in Rat's hepatocytes.

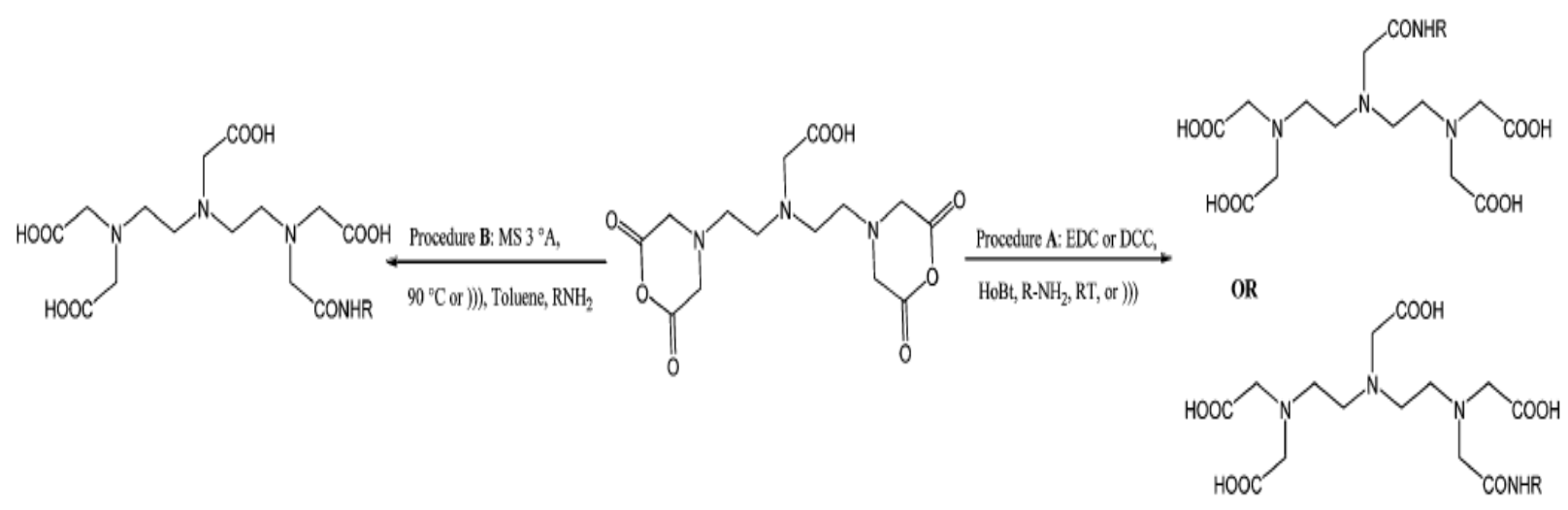

Figure 2. Procedures A and B described for the exclusive synthesis of DTPA-mono-amide while using DCC or EDC or toluene/molecular sieve at presence or absence of ultrasound irradiation [13]

\section{Material and methods}

\subsection{Material}

Hexanes, 1-hexanol, cyclohexane, pyridine, diethylenetriamine pentaacetic acid dianhydride, methanol, and aqueous $\mathrm{NH}_{4}{ }^{+} \mathrm{OH}^{-}$were purchased from Aldrich. $N, N$-dimethylformamide (DMF), $N, N$ dicyclohexylcarbondiimide $\quad$ (DCC), anhydrous ethanol, and acetone were analytical grade without further purification. Double distilled water was used. Dialysis bag with $5 \mathrm{KD}$ cut off was obtained from spectrum.

\subsection{Characterization}

Melting points were determined on a Kofler hot stage apparatus and are uncorrected (C. Reichert, Vienna, Austria). The FT-IR spectra were recorded on a Nicolet 550 instrument. $1 \mathrm{H}$ and 13C NMR spectra were measured on a Varian FT-400 unity plus (400 MHz for ${ }^{1} \mathrm{H}$ and $100 \mathrm{MHz}$ for ${ }^{13} \mathrm{C}$ ) spectrometer and also a Bruker $500 \mathrm{MHz}$ spectrometer (Brucker, Rheinstetten, Germany) spectrometer with tetramethyl silane as an internal standard. Chemical shifts are given in d. EIMS spectra were recorded on a Finnigan 
MAT EITSQ-70 eV (Finnigan, USA) and also on Agilent Technology (HP), 5973 Network Mass. FT-IR spectra was examined by FT-IR spectroscopy (Thermo-Nicolet, NEXUS 870, Waltham, MA, USA.Silica gel $60 \mathrm{~F}_{254}$ precoated plates (Merck) were used for TLC. The purity of the synthesized compounds was confirmed by thin-layer chromatography (TLC) using various solvents of different polarities. The morphology was studied by using an inverse phase-contrast microscope (Wilovert ${ }^{\circledR}$, Hund Wetzlar, Wetzlar, Germany. Sonication was performed using a Sartorius Ultrasonic-Homogenizer LABSONIC_P230 V/50 Hz. Copper concentration was determined by Flame atomic absorption spectrophotometer (PERKIN ELMER ). The $\mathrm{pH}$ measurements of all solutions were done with $\mathrm{pH}$ meter And Cell debris were collected by centrifuge model Sigma 316PK(Germany).

\subsection{Synthesis of DTPA-dianhydride}

The DTPA-dianhydride (DTPA-DA) synthesis procedure has been previously described[7,13]. Briefly, DTPA (3 g) was dissolved in dimethyl sulfoxide $(10 \mathrm{ml})$, acetic anhydride $(20 \mathrm{ml})$, and pyridine $(3 \mathrm{ml})$ as a base under anhydrous conditions. The reaction mixture was heated at $65^{\circ} \mathrm{C}$ for one day. Subsequently, the reaction mixture was cooled, filtered, and washed twice in acetic anhydride and anhydrous diethyl ether. The residue was dried to a constant weight under vacuum $(52 \mathrm{kPa})$ at $40^{\circ} \mathrm{C}$, and a white powder was produced with a yield of $93 \%$ (see Fig. 2).

\subsection{Activation of D-glucosamine hydrochloride}

D-glucosamine hydrochloride was neutralized using excess amounts of a base, such as aqueous sodium bicarbonate [13] The reaction was stirred for at least 30 minutes, then filtered, and ascorbic acid added to the solution. The solution was then immediately lyophilized, and a mild yellowish powder (D-glucosamine, base) was obtained, with a yield of $97 \%$.

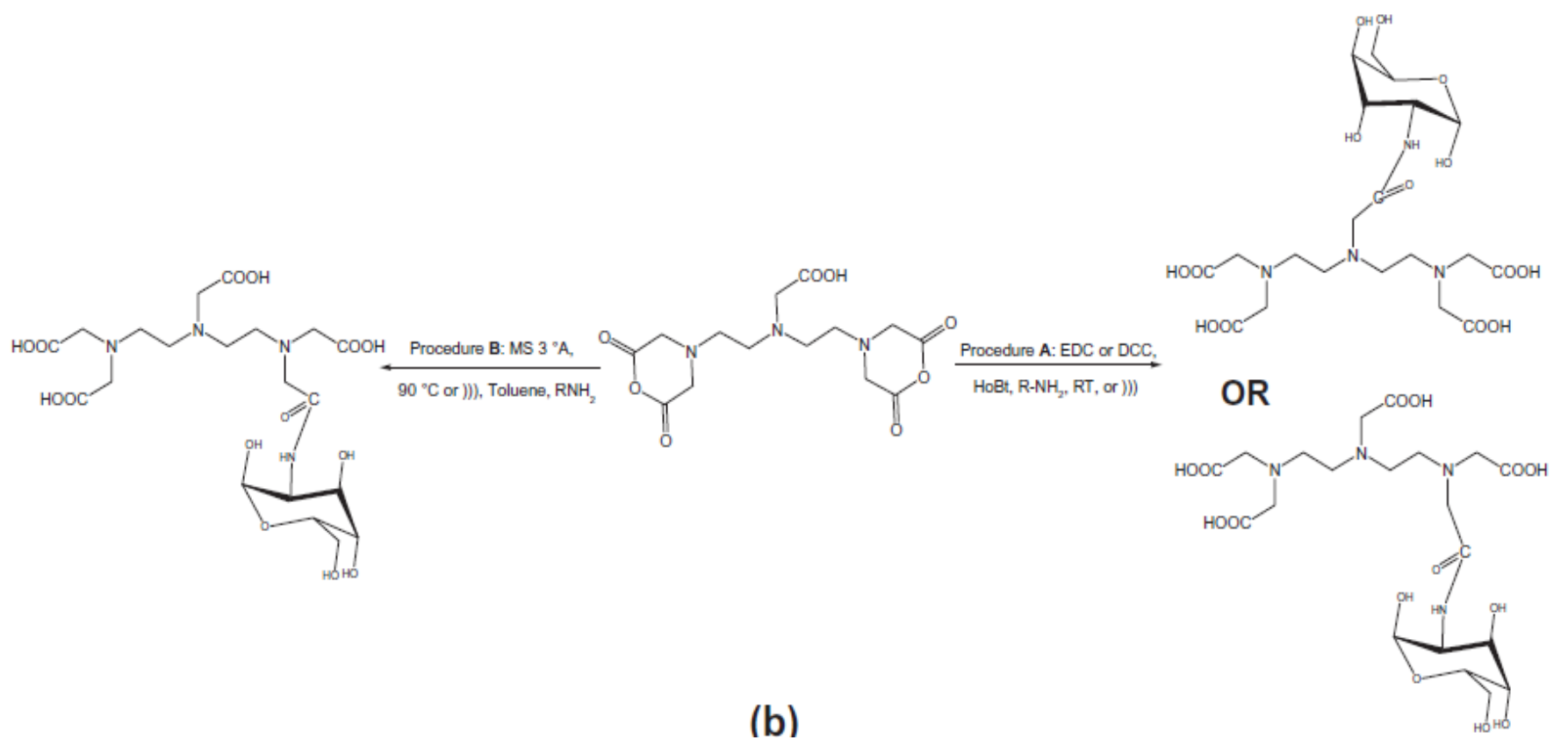

Figure 3: General procedure for synthesis of DTPA-DG [7]

\subsection{Synthesis of DTPA-DG}

DTPA-DG synthesis procedure has been previously described [7,13]. Briefly, DTPA-DA (1 mmol) was dissolved in anhydrous DMF, and then $N, N$ '-dicyclohexylcarbodiimide (DCC) (1.1 mmol), 1-hydroxy-benzotriazole (HOBT) (0.6 mmol), triethylamine $(0.2 \mathrm{ml})$, and activated molecular sieve (100 $\mathrm{mg})$ were added to the 
medium. The reaction mixture was stirred at room temperature for at least 30 minutes. The reaction solution was subsequently heated to $90^{\circ} \mathrm{C}$, and D-deoxy-glucosamine (DG) (0.333 mmol) was added. After stirring for an additional $24 \mathrm{~h}$ at $90^{\circ} \mathrm{C}$, the reaction mixture was cooled at room temperature, diluted with water $0.2 \mathrm{~mL}$ and methanol $5 \mathrm{ml}$, and then filtered. A clear solution was collected.The crystallized white DTPAmonoamide (DTPA-DG) was obtained by adding diethyl ether $(0.5 \mathrm{ml})$ to the solution and subsequently filtered and finally dialyzed (cut off $500 \mathrm{Da}$ ) against distilled water for a period of 24 $\mathrm{h}$. The remaining solution was removed from the dialysis bag and then lyophilized. A pure white powder was obtained [7,13]. (Fig.3)

\subsection{Gel chromatography and TLC}

The sample mixture were separated based on their molecular size and shapes by Gel permeation chromatography (GPC)[15]. Before experimental, the stationary phase (sephadex G15) was saturated by PBS. [16-21]. The mixture in 2-4 $\mathrm{ml}$ was added to the column (high=30 $\mathrm{Cm}$, Width $=3 \mathrm{Cm}$ ). Each 20 elution drops was collected and placed in a separate tube (13 tubes). Then each tube was checked by TLC to find the DTPA-DG. As shown in fig.3 there were 2 standard spots (DTPA and DTPA -DG). The TLC plate was placed in the chamber so that the $\operatorname{spot}(s)$ of the sample do not touch the surface of the elutant in the chamber. The solvent $(7 \mathrm{ml}$ chloroform and $3 \mathrm{ml}$ methanol) moves up the plate by capillary action, were meet the sample mixture and carries it up the plate (elutes the sample). When the solvent front reaches no higher than the top of the filter paper in the chamber, the plate should be removed (continuation of the elution will give a misleading result) and dried .The retention factors $\left(\mathrm{R}_{f}\right)$ were defined as the ratio of the distance traveled by the substance to the distance traveled by the solvent (FIG.4). Tubes containing only DTPA-DG were chosen for the lyophilization step and stored for next subsequent experiments (FIG.4) [20, 21].

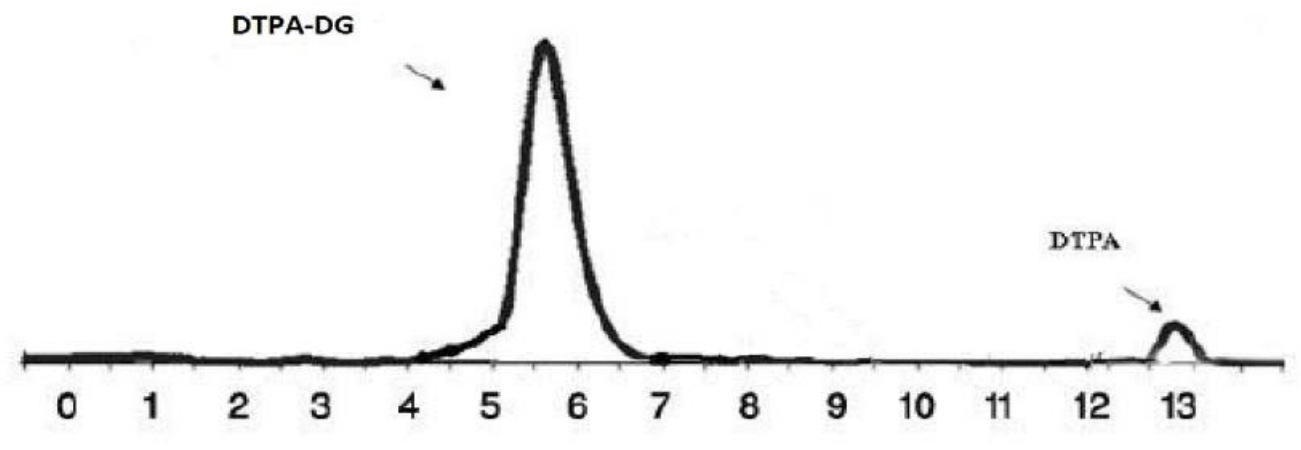

(ml)

Figure 4: Demonstrative purification steps by sephadex G-25 fine system.

\subsection{Wilson's model cells preparation}

Human HepG2 cells ( $10^{6}$ cells) were growth in Minimum Essential Medium (DMEM) with glutamine. The cell culture were supplemented with FBS (10\%), penicillin G (2000 U/ml) and streptomycin $(200 \mathrm{U} / \mathrm{ml})$. During the passage, rinse cell with medium thrice. Then cells were growth in medium with $0.5 \mu \mathrm{mol} \mathrm{CuSO}_{4}$ (physiologic concentration) or $100 \mu \mathrm{mol} \mathrm{CuSO}_{4}$ (pathologic concentration) for $48 \mathrm{~h}$ to reach WD cell model [19-21].

\subsection{Preparation of cell culture}

HepG2 are adherent, epithelial-like cells growing as monolayers and in small aggregates. For preparation of this cell culture we were used Eagle's Minimum Essential Medium (DMEM and RPMI1640 also works well) supplemented with 
10\% FBS. Discard culture medium every 2-3 days. To passage cells, briefly rinse cell monolayer with $1 \times$ PBS twice and add prewarmed $\left(37^{\circ} \mathrm{C}\right) 0.05 \%$ Trypsin-EDTA solution for 5 - 7 minutes. Once cell layer is dispersed (5$7 \mathrm{~min}$ at $37^{\circ} \mathrm{C}$ ) deactivate Trypsin by adding equal volume of complete growth medium with $10 \%$ FBS. To avoid clumping don't agitate the cells by shaking the flask while waiting for the cells to detach. Split cells 1:4 every 3 days (recommended), or 1:8 every 6 days.Cultures should be incubated at $37^{\circ} \mathrm{C}$ in humidified atmoshere with $5 \% \mathrm{CO}_{2}$ [24-25].

\subsection{MTT assay}

Cell viability was measured by The MTT assay.[7,26-30].The HepG2 cells were incubated with the assorted concentration $(0.125,0.25$, and $0.5 \mathrm{mg} / \mathrm{ml}$ ) of DTPA-DG for 24-48h, afterwards the supernatant of the Hepg2 cells was removed. MTT answer was additional to every well of the plates at a final concentration of zero. $5 \mathrm{mg} / \mathrm{mL}$, and also the cells were incubated for a further four $\mathrm{h}$. Thereafter, remaining solution was removed, the cells were lysed, and also the dye was dissolved in a hundred $\mu$ l dimethyl sulfoxide. The plates were unbroken within the dark for one hour so as to be prepared for spectrophotometric determination. the quantity of absorption in every well was calculated mistreatment an automatic microplate reader at $570 \mathrm{~nm}$. The data were compared with untreated control cultures.

\subsection{Intracellular measurement of copper concentration and DTPA-DG potency assay by atomic absorption}

DTPA-DG and D-penicillamine at different doses were injected on separate plates containing $10^{6}$ cells and the experiments were repeated for at least three times for each dose. After incubation time of $24 \mathrm{~h}$ DTPA-DG and cells, the cells have been broken by grinding glass beads in a $50 \mathrm{ml}$ tube at $100^{0 \mathrm{C}}$ by a $45 \mathrm{~Hz}$ electric motor for 20 minutes [31-32]. The seeds and disrupted cells were removed by centrifugation; Supernatant solution was removed for copper assay with Atomic Absorption spectroscopy. There were 2 control groups: Positive control containing Wilson's cell model (WD) the intracellular $\mathrm{Cu}$ concentration was about $50 \mathrm{ppm}$. Untreated cells and medium were negative control group.

\subsection{DTPA-DG EC50 calculation}

The term half maximal effective concentration $\left(\mathrm{EC}_{50}\right)$ refers to half concentration of a drug which causes to decrease intracellular copper concentration to $50 \%$ of ground state (WD cell model) [33-34].In this study EC50 was calculated based on the linear model curve with Pharm ${ }^{\mathrm{R}}$ statistical software.

\subsection{Animals}

Frothy male and female rats (mean weight 90 $\pm 20 \mathrm{~g}$ ) were used for this study. The animal were provided by Iran Pasteur Institute laboratory animal center. The animal experiments were performed in accordance with the recommendations of the Declaration of Helsinki and the internationally accepted principles for use of experimental animals, and they were confirmed by the local ethics committee of our institution as well. The animals were drived into four groups, 10 animals in each. They were divided into 4 groups as follows:

\section{Group A: placebo}

Group B: $5 \mu \mathrm{g}$ (15 times the therapeutic dose) for

2 weeks. Volume of administered was $230 \mu \mathrm{l}$

Group C: $10 \mu \mathrm{g}$ (30 times the therapeutic dose) Group C:25 $\mu \mathrm{g}$ (45 times the therapeutic dose)

After 14 days, the rats were sacrificed with cervical dislocation. Following sacrifice, a portion of liver, spleen, ganglion and kidny were removed for following pathologic experiment:

- FG-focal granuloma

- MG-multiple granoloma

- N-Necrosis

- EO-polymorphic nuclar

- EH-extra-medullary hemotopoesis

The toxicity experimentals were measured according to these protocols:

1-International committee for harmonization of biological compounds (ICHBC)

2- committee of proprietors of mwdical products(CPMC)

Volume and dosage of administration were according tables 2 and 3. 


\subsection{Statistical analysis}

Data interpretations were performed by SPSS 19 software using One way ANOVA with post hoc TUKEY test and the data comparison covering $\mathrm{P}<0.05$ was considered significant.

\section{Result and discussion}

\subsection{Synthesis}

The DTPA-mono amid is synthesized in temperature over $90^{\circ} \mathrm{C}$ with high yields and in pure form, as confirmed by FTIR, Mass and HNMR [13].

\subsection{TLC}

As shown in table 1 , and according to TLC results, the first four tubes showed no growth in a spot regarding not any materials. Tube numbers 5 and 6 were showed TLC spots with $\mathrm{Rf}=0.56$. These were indicated that these samples were polar and included DTPA-DG. $\mathrm{Rf}_{9}=0.91$ and $\mathrm{Rf}_{13}=0.96$ were shown these samples were contained unconjugated-DTPA. Based on the gel filtration rules high molecular weight compound is exit from the column faster than those of lower molecular weight materials and based on the explained reasons (different $\mathrm{R}_{\mathrm{f}}$ and rapid exist from the column) tubes 5 and 6 were identified to contain DTPA-DG.

Table 1: TLC results

\begin{tabular}{|l|l|l|}
\hline Tube & Rf & Spots \\
\hline 1 & - & - \\
\hline 2 & - & - \\
\hline 3 & - & - \\
\hline 4 & - & - \\
\hline 5 DTPA-DG & 0.56 & Detect \\
\hline 6 DTPA-DG & 0.56 & Detect \\
\hline 7 & - & - \\
\hline 8 & - & - \\
\hline 9 & 0.91 & Detect \\
\hline 10 & - & \\
\hline 11 & - & \\
\hline 12 & - & \\
\hline 13 & 0.96 & Detect \\
\hline St.DTPA & 0.95 & Detect \\
\hline St. DG & 0.91 & Detect \\
\hline
\end{tabular}

Table 2: The female rat's weight changes

\begin{tabular}{llll} 
Group & Day1 & Day7 & Day 14 \\
\hline A & $90.6 \pm 4.72$ & $135 \pm 5.03$ & $158.8 \pm 4.44$ \\
B & $90.40 \pm 5.64$ & $137.6 \pm 3.21$ & $155.4 \pm 8.38$ \\
C & $90.40 \pm 4.04$ & $132.8 \pm 9.71$ & $149.6 \pm 5.77$ \\
D & $90.60 \pm 5.64$ & $132.8 \pm 8.32$ & $147.8 \pm 3.42$ \\
\hline
\end{tabular}

Table 3: The male rat weight changes

\begin{tabular}{llll} 
Group & Day1 & Day7 & Day 14 \\
\hline A & $100.6 \pm 2.88$ & $156.2 \pm 7.28$ & $203.2 \pm 14.43$ \\
B & $100.2 \pm 3.27$ & $156.2 \pm 3.78$ & $193.8 \pm 16.5$ \\
C & $100.6 \pm 2.61$ & $159 \pm 4.47$ & $204.2 \pm 7.76$ \\
D & $100.4 \pm 3.13$ & $165 \pm 6.4$ & $215 \pm 8.86$ \\
\hline
\end{tabular}

\subsection{MTT assay}

The MTT test was performed on Hep G2 cell types, The 24-hour exposure of DTPA-DG did not show any significant toxicity on these cell types, but continued exposure for 48 hours showed very significant $(P, 0.05)$ and powerful anticancer potency for DTPA-DG ( Figs-5-9.).
The unlabeled ligands (DTPA) were also demonstrated to be safe.

3.4 Intracellular Measurement of copper concentration and DTPA-DG potency assay by atomic absorption 
The effect of DTPA-DG and DTPA on intracellular copper were shown in Fig 9 and Fig 10. DTPA-DG with different doses from 10 to $100 \mu \mathrm{g} / \mathrm{ml}$ were administered on WD cell models could be conjugated with intracellular copper and were decreased intercellular copper accumulation $(p<0.05)$. As shown in Fig 10 DTPA wasn't shown any significant alteration in copper concentration. The result was indicated that DTPA has negative charge so could not able to cell penetrate but DTPA-DG has very good potency to decrease copper concentration (significant decrease in intercellular copper concentration) because DTPA-DG is well shown cell penetration.Figure.8 was Illustrated of comparative effects of DTPA-DG and control groups on intercellular copper concentration. Fig.9: Illustration of the high dose comparative effects of Nano-scale of DTPA with Dpenicillamine. Results were indicted that DTPADG has perfect potency to decrease intracellular copper accumulation.

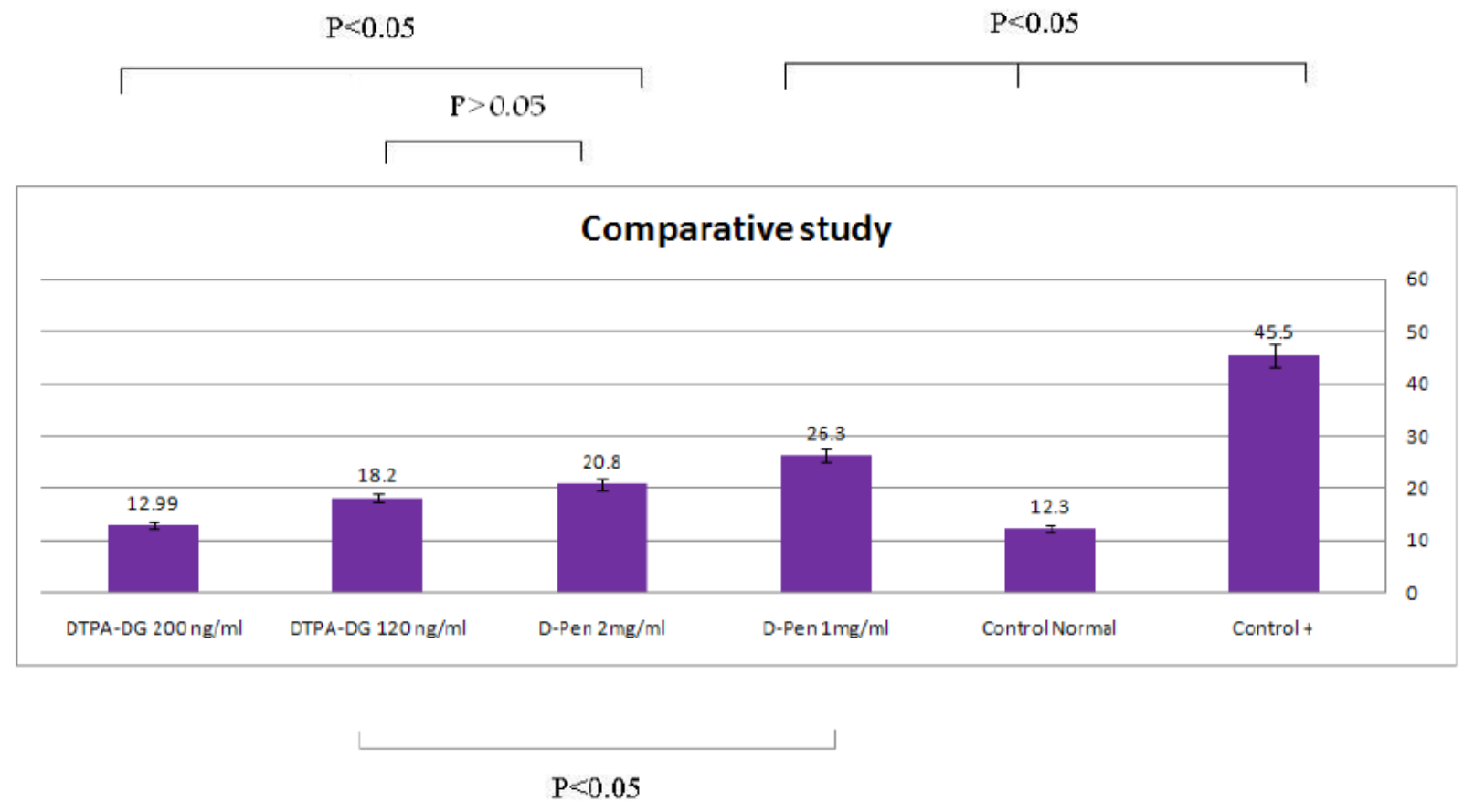

Figure 5: MTT results before and after nano-scaled DTPA exposure to HepG2 cells. No toxic effect was observed. (DTPA-DendrimerG2 conjugate different doses were assigned as numbers 1, 2, 3, 4 which equals to 50, 100, 150 and $200 \mu \mathrm{g} / \mathrm{ml}$ respectively, DTPA concentration was $200 \mu \mathrm{g} / \mathrm{ml}$ )

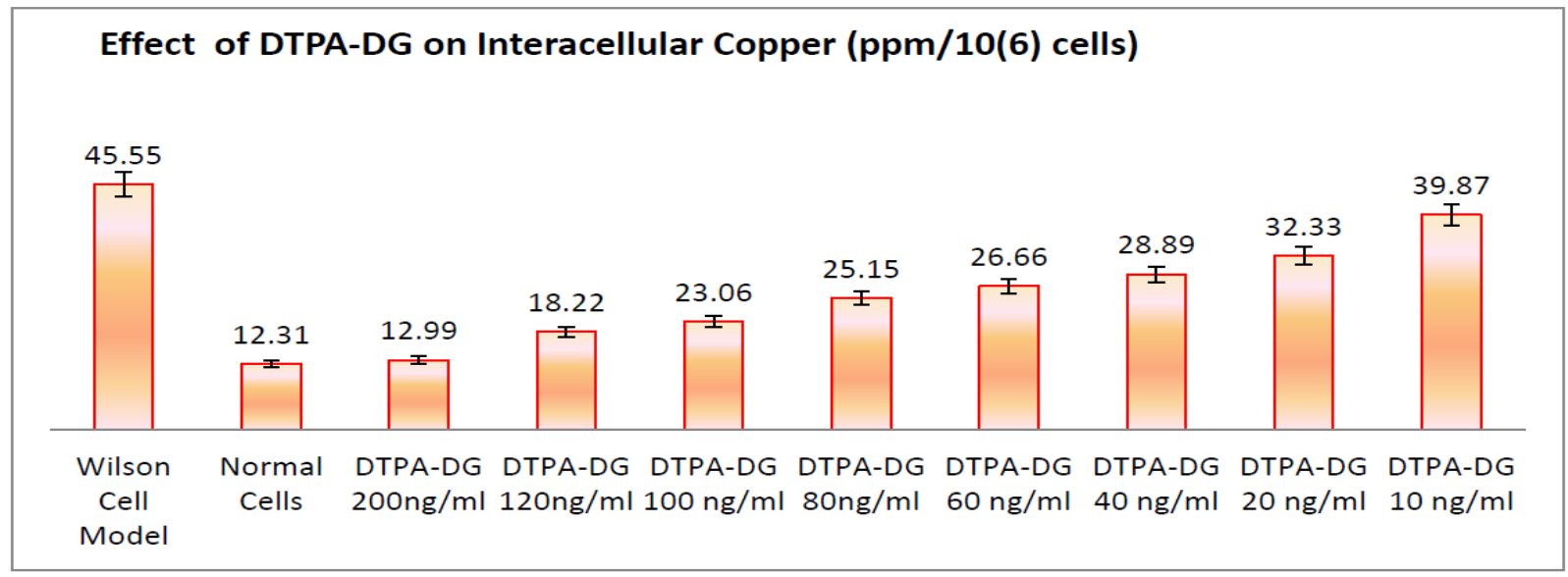

Figure 6: Effects of different doses of DTPA-DG on intercellular copper concentration. 


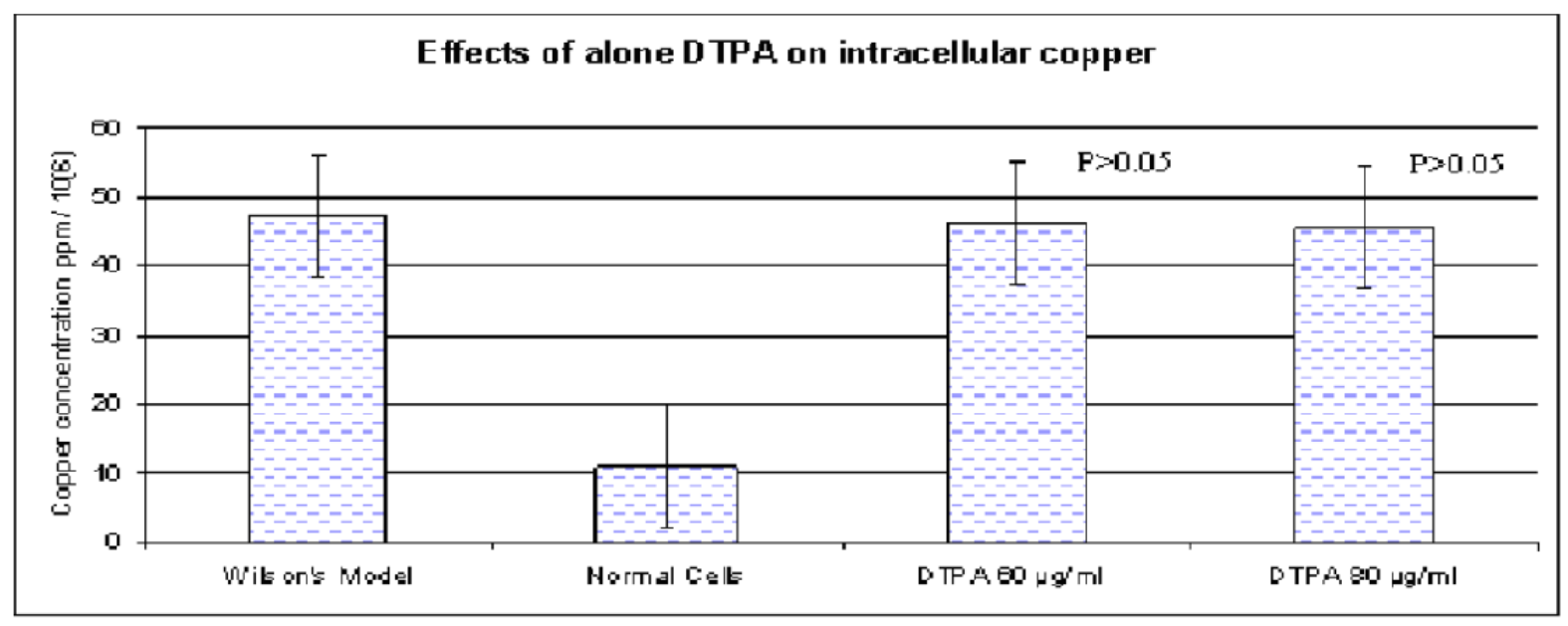

Figure 7: Effects of different doses of DTPA on intercellular copper concentration.

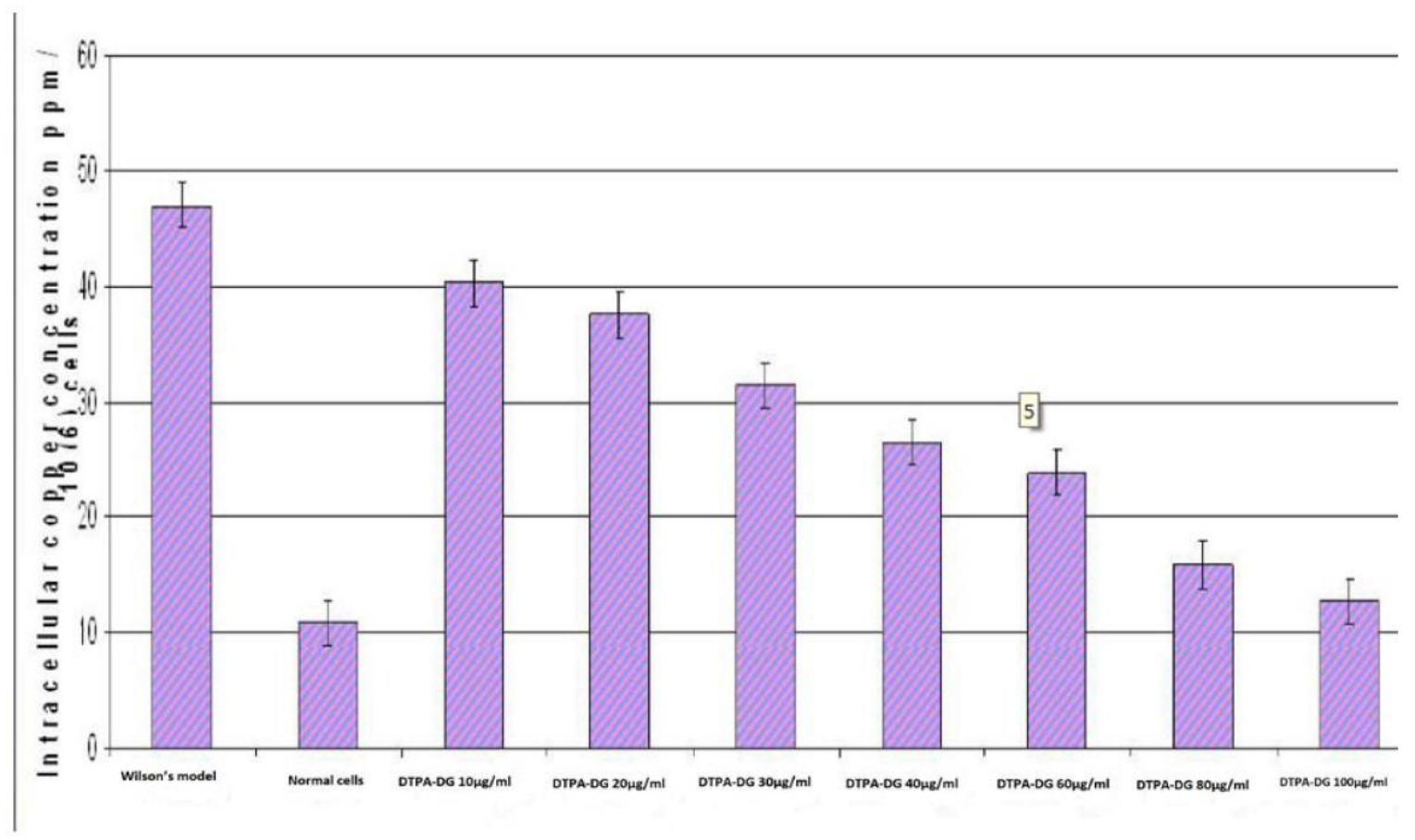

Figure 8: Illustration of comparative effects of DTPA-DG and control groups on intercellular copper concentration

\subsection{EC50 Calculation}

Figure 10 was shown EC 50 curve. This curve was indicated the relation between different doses of DTPA-DG (X) and copper concentration (Y). We were calculated EC50 as below:

EC50 WAS One dose that could decreased the Wilson Cell Model Copper level from 45.55 ppm to $50 \%$ (22.775 ppm).As a result DTPA-DG
EC50 was calculated based on the below linear model:

$Y=-0.116 x+35.10$

$\mathrm{y}=22.775=-0.116 \mathrm{x}+35.10$

$0.116 \mathrm{x}=35.10-22.775=12.325$

$\mathrm{X}=\mathrm{DTPA}-\mathrm{DGEC} 50=106.25 \mathrm{ppm}$

$106.25 \mathrm{ppm}$ is apparently much less that Dpenicillamine EC50 and based on its safety and 
powerful effects it can propound as new WD

alternative therapy in near future.

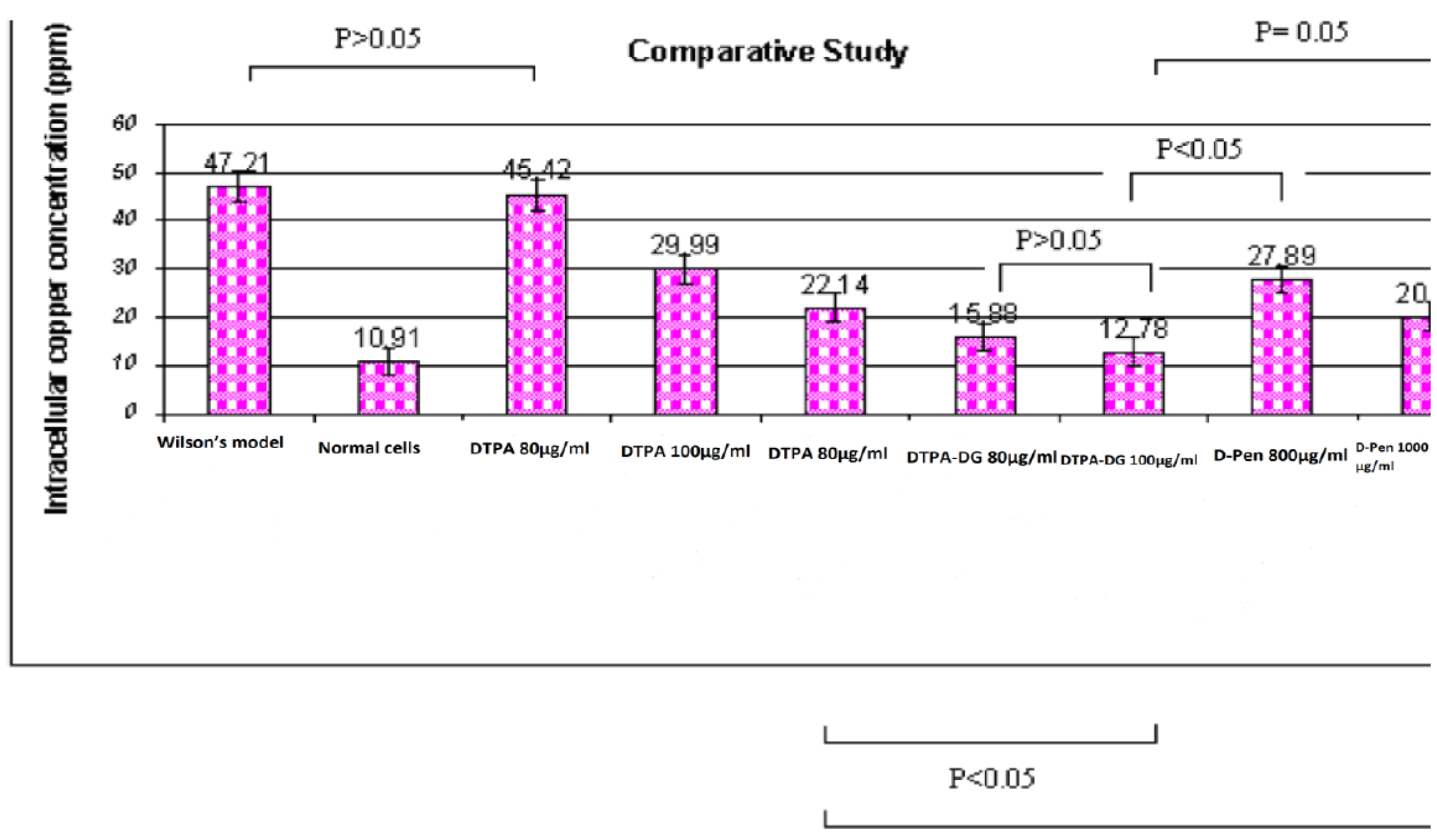

Figure 9: Illustration of the high dose comparative effects of Nano-scale of DTPA with D-penicillamine

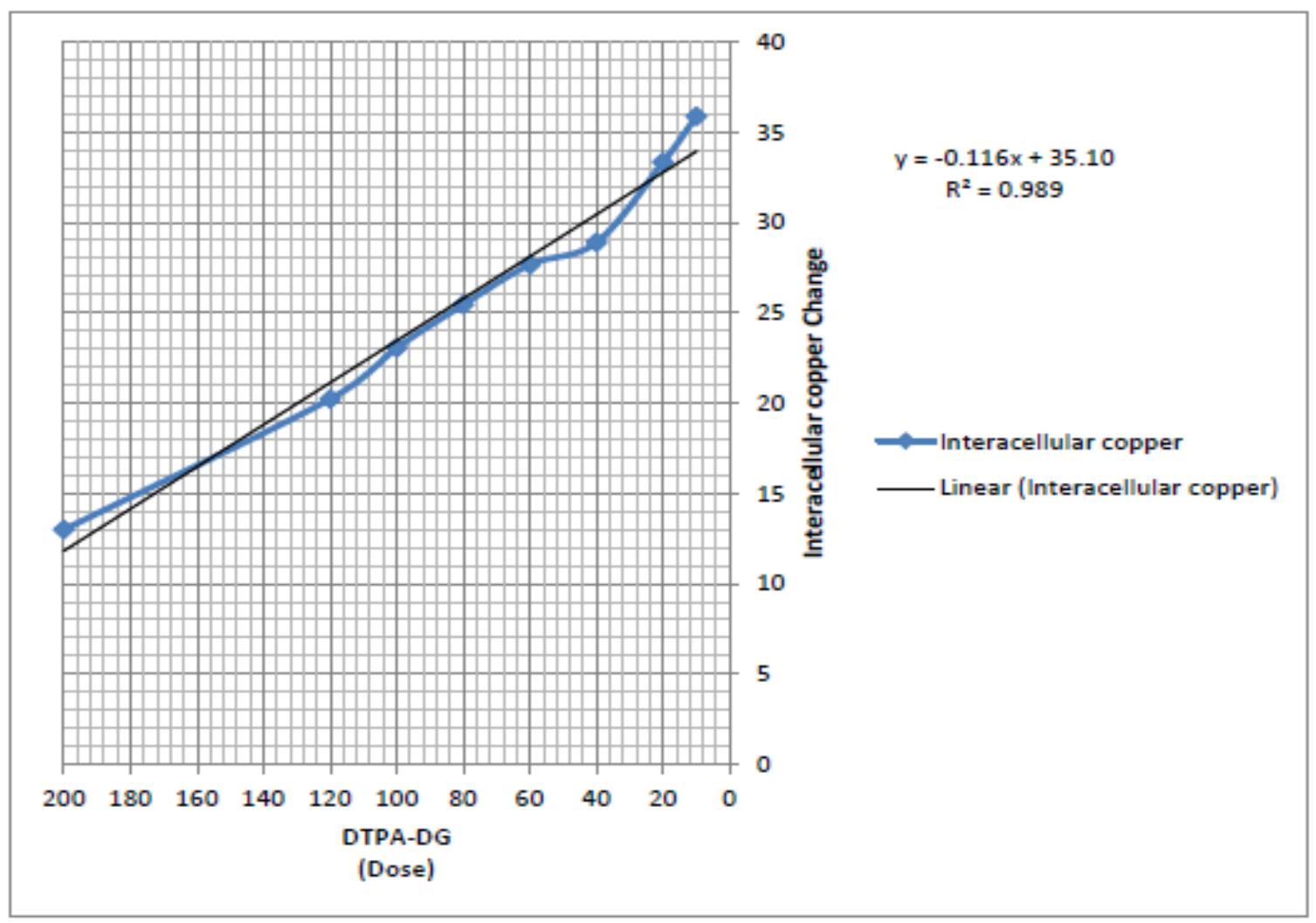

Figure10: EC50 demonstrative cure which is the DTPA-DG dose curve that determines the 50\% potency in reduction of copper is deposited for the generated DTPA-DG. (Colored line is the obtained original data curve and black line is the excel calculated curve which is completely correlated with the original data) 


\subsection{Animals}

Tables 2 and 3 were shown the female and male rat's weight changes, respectively. The result were shown that no tissues toxicity.

The result of pathologic study on kidney and liver after 2 weeks after DTPA-DG injection were shown that no toxicity indicated on liver and kidney. (Fig. 11 and Fig. 12).
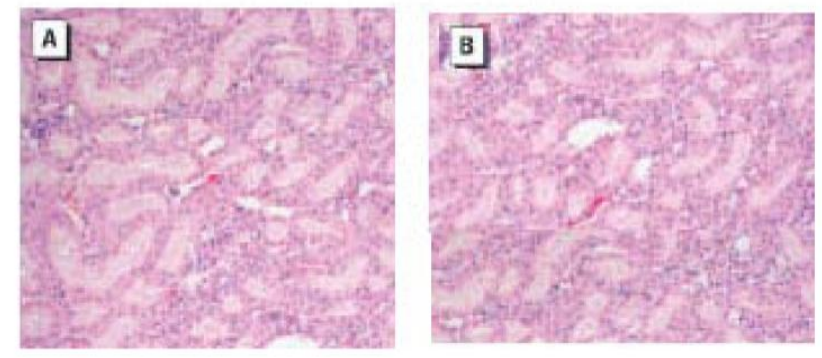

Figure 11: pathologic study on liver
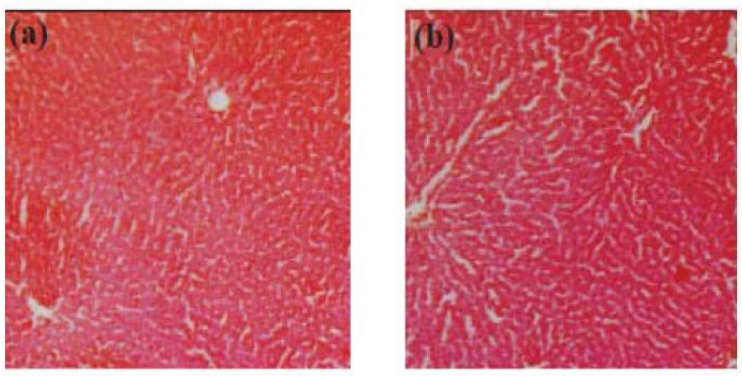

Figure 12: phathologic study on kidney

\section{Conclusions}

In summary, we have designed and characterized DTPA-DG with extraordinary ability to decrease intracellular copper accumulation. Its utility as a novel chelating agent for intracellular copper overload has been clearly demonstrated in vitro. We have also shown that DTPA-DG has very potent copper chelating effect without any significant toxicity on HepG2 WD. The microscopic images have shown no change of the Hep G2 cell morphology after the administration of DTPA-DG. EC50 of DTPA-DG $(106.25 \mu \mathrm{g} / \mathrm{ml})$ is apparently much less than D-penicillamine So due to its safety and powerful effects, it can be propounded as a new alternative therapy for WD alternative therapy in near future. We are currently evaluating the potential of utilizing this class of nano-materials as target-specific chelating agents for intracellular treatment of metal overload. It it seems that this class of agents has a positive future in clinical practice.

\section{Acknowledgement:}

We acknowledge financial support from Tehran University of Medical Sciences and Pasteur Institute of Iran. The authors are grateful to thank all technicians who provided support during the course of research.

\section{References}

1- Bibudhendra Sarkar, treatment of Wilson and menkes disease. Chem .Rev. 1999,99, 25352544

2- Lauren E. Scott and chris orvig. Medical inorganic chemistry approaches to passivation and removal of aberrant metal ions in desease, chem. Rev . 2009, 109,4885-4910. DOI: $10.1021 / \mathrm{cr} 9000176$

3- Scott, L. E.; Orvig, C., 'Medicinal Inorganic Chemistry Approaches to Passivation and Removal of Aberrant Metal Ions in Disease', Chemical Reviews, 2009, 109(10), 48854910. DOI: $10.1021 / \mathrm{cr} 9000176$

4- Ole. Anderson. Principles and recent developments in chelation treatment of metal intoxication, chem.. rev. 1999, 99 , 26832710. DOI: $10.1021 / \mathrm{cr} 980453 \mathrm{a}$

5- Haririan I, Shafiee-Alavidjeh M, Khorramizadeh MR, Shafiee- Ardestani M, Zarei-Ghane Z, Namazi H. Anionic linearglobular dendrimer- cis-platinum [II] conjugates promote cytotoxicity in vitro against different cancer cell lines. Int $J$ Nanomedicine. $\quad 2010 ; 5: 63-75 . \quad$ DOI: 10.2147/IJN.S8595

6- Kainthan RK, Hester SR, Levin E, Devive DV, Brooks DE. In vitro biological evaluation of 
high molecular weight hyperbranched polyglycerols. Biomaterials. 2007;28:45814590.

DOI:10.1016/j.biomaterials.2007.07.011

7- Shafiee- Ardestani M,Amanlou M., Gd3+DTPA-DG: novel nanosized dual anticancer and molecular imaging agent, International Journal of Nanomedicine 2011:6 747-763. doi: $10.2147 /$ IJN.S17648

8- Werner EJ, Botta M, Aime S, Raymond KN. Effect of a mesitylene-based ligand cap on the relaxometric properties of $\mathrm{Gd}[\mathrm{III}]$ hydroxypyridonate MRI contrast agents. Contrast Media Mol Imaging. 2009;4: 220229. DOI: $10.1002 / \mathrm{cmmi} .281$

9- Raymond KN, Pierre VC. Next generation, high relaxivity gadolinium MRI agents. Bioconjug Chem. 2005;16:3-8. DOI: 10.1021/bc049817y

10- Sun YY, Chen Y. Cancer drug development using glucose metabolism radiopharmaceuticals. Curr Pharm Des. 2009;15:983-987. ISSN : 1381-6128

11- Marshall S, Nadeau O, Yamasaki K. Glucosamine-induced activation of glycogen biosynthesis in isolated adipocytes: Evidence for a rapid allosteric control mechanism within the hexosamine biosynthesis pathway. $J$ Biol Chem. 2005;280:110-118. DOI 10.1074/jbc.M413499200

12- Schechter NR, Erwin WD, Yang DJ, et al. Radiation dosimetry and biodistribution of [99 m]Tc ethylenedicysteine-deoxyglucose in patients with non-small-cell lung cancer. Eur J Nucl Med Mol Imaging. 2009; 36:15831591. doi: 10.1007/s00259-009-1135-8

13- Shafiee-Ardestani M, Jabbari-Arabzadeh A, Heidari Z, et al. Novel and facile methods for the synthesis of DTPA-mono-amide; a new completely revised strategy in radiopharmaceutical chemistry. J Radioanal Nucl Chem. 2010;283:447-455. DOI: 10.1007/s10967-009-0414-y

14-Yang DJ, Kim CG, Schechter NR, et al. Imaging with $99 \mathrm{mTc}$ ECDG targeted at the multifunctional glucose transport system: Feasibility study with rodents. Radiology. 2003;226:465-473.

doi:
15- Porath J. and P. Flodin. chromatography gel

- filtration Nature(1959). 183, 1657 doi: $10.1038 / 1831657 \mathrm{a} 0$

16- Vaughan.M. F. chromatography gel filtration, Nature(1960). 188, 55

17- Moore.J. C. .1. Polymer Sci. A2 chromatography gel - filtration, (1964), 835

18- Determann.H. Gelchromatographie, Springer Verlag, Berlin, Heidelberg, New York, (1967). DOI: 10.1002/ange.19680801232

19- FRIED,B. J. SHERMA: Thin-Layer Chromatography, Fourth Edition,revised and expanded, Marcel Dekker Inc., New York Basel, (1999), 499 pages, ISBN 0-8247-02220 .

20- http://orgchem.colorado.edu/hndbksupport/ TLC/TLC.html

21 - http://www.chromatography-online.org/

22- Odlyha,. M. C.Theodorakopoulos, J. de Groot, L. Bozec, M.Horton, Thermoanalytical (macro to nanoscale) techniques andnoninvasive spectroscopic analysis for damage assessment ofparchment, in: Improved Damage Assessment of Parchment, IDAPEC Research report No. 18, ISBN 978-92-7005378-8), 73-85.

23- Larsen (Ed.). R., Improved Damage Assessment of Parchment,IDAP EC Research report No. 18, ISBN 978-92-70-05378-8.

24- Wodicka, L., Dong, H., Mittmann, M., Ho, M.H. \& Lockhart, D.J. Genome-wide expression monitoring in Saccharomyces cerevisiae. Nat Biotechnol .(1997)15, 13591367. doi: $10.1038 /$ nbt1297-1359

25- Newton, D.J., Wang, R.W. \& Lu, A.Y. Cytochrome P450 inhibitors. Evaluation of specificities in the in vitrometabolism of therapeutic agents by human liver microsomes. Drug Metab Dispos(1995). 23, $154-158$

26- Slater, T. et al. Biochem. Biophys. $\operatorname{Acta}(1963), 77: 383$.

27- van de Loosdrecht, A.A., et al. J. Immunol. Methods (1994), 174: 311-320

28-Alley, M.C., et al. Cancer Res. (1988), 48: 589-601,

29- Fastrez, J. Phage Lysozymes In: Lysozymes-model enzymes in biochemistry and biology, $\underline{10.1148 / \text { radiol.2262011811 }}$ 
Pierre Jollès editor, Birkhäuser,( 1996), pp. 35-64. ISBN: 3-76435121-7

30- Scholar, E. M., Pratt W. B. The antimicrobial drugs, Oxford University Press US, 2nd ed.,( 2000), pp. 61-64.

31- Skoog .D. A. D. M. West - F. J. Holler: Fudamentals of AnalyticalChemistry (1992). ISBN: 0030148138

32- Kenkel J.: Analytical Chemistry for Technicians (1994). ISBN: 0873719662, 9780873719667

33- Cheng Y, Prusoff WH "Relationship between the inhibition constant (K1) and the concentration of inhibitor which causes 50 per cent inhibition (I50) of an enzymatic reaction". Biochem Pharmacol (1973). 22 (23): 3099-108. ISSN: 0006-2952 DOI: $\underline{10.1016 / 0006-2952(73) 90196-2}$
33- Cheng Y, Prusoff WH "Relationship between the inhibition constant (K1) and the concentration of inhibitor which causes 50 per cent inhibition (I50) of an enzymatic reaction". Biochem Pharmacol (1973). 22 (23): 3099-108. ISSN: 0006-2952 DOI: 10.1016/0006-2952(73)90196-2

34- Robinson, SF; Marks, MJ; Collins, AC "Inbred mouse strains vary in oral selfselection of nicotine". Psychopharmacology

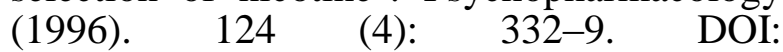
10.1007/BF02247438

35- http:// www.wikipedia.org/ 